\title{
Presentation Of Comparative Data for Transportation Planning Studies
}

\author{
Joseph W. Guyton, HNTB Corporation
}

\begin{abstract}
Clear, yet detailed, presentations of transportation planning data to lay groups as well as to technical groups is becoming more and more of a necessity in the planning process. The transportation professional is faced with the challenge of presenting technical analyses in a manner permitting others to make their own, sound, choices. Increasingly, the choice of a preferred alternative involves both technical aspects and seemingly intangibles or quality aspects. Technical analyses often seem to press in one direction while other considerations give a different perspective on the preferred solution.

Peer groups as well as administrators of programs are faced with the need to quickly understand complex planning relationships. Presentation of technical information in understandable terms has become increasingly critical to the decision making process. Professionals develop significant quantitative data to compare and contrast alternatives under investigation, and there is a need to render the results comprehensible for those making recommendations and those making decisions.

Is the oft-used analysis technique of measuring the effectiveness of various alternatives and screening the results becoming inappropriate? Can a process be developed to permit comparisons based on quantitative data and on qualitative measures? Is there a way to permit individuals to use transportation planning data with individualized weightings and judgments to compare alternatives? Can the transportation professional improve the communication channels while maintaining objectivity and comprehensiveness? These are some of the questions which are in need of attention.

Policy statements by FTA and by ITE highlight several approaches to the comparison of alternatives and the selection of measures of effectiveness for those comparisons. This paper addresses the application of simple but effective comparison techniques designed to provide high flexibility to individuals in comparing alternatives.

The presentation of technical results from transportation studies in spreadsheet formats gives the basis for objective comparisons while incorporating the variety of viewpoints on the relative merits of the selected measures of effectiveness. This paper explores the screening techniques used in recent studies for ISTEA High Priority Corridors and feasibility studies and illustrates how the use of a matrix analysis technique has applications in many studies. These approaches were used to assist technical and policy committees in the selection of a preferred corridor. The intent of the paper also is to place the approach in the proper context for various study applications.
\end{abstract}

Clear, yet detailed, presentations of transportation planning data to lay groups as well as to technical groups is becoming more and more of a necessity in the planning process. The transportation professional is faced with the challenge of presenting technical analyses in a manner permitting others to make their own, sound, choices. Increasingly, the choice of a preferred alternative involves both technical aspects and seemingly intangibles or quality aspects. Technical analyses often seem to press in one direction while other considerations give a different perspective on the preferred solution.

Peer groups as well as administrators of programs are faced with the need to quickly understand complex planning relationships. Presentation of technical information in understandable terms 
has become increasingly critical to the decision making process. Professionals develop significant quantitative data to compare and contrast alternatives under investigation, and there is a need to render the results comprehensible for those making recommendations and those making decisions.

At times, the technical analyses (on one hand) and (on the other) making the choice of a preferred alternative seem to be at odds with each other. Making a choice with multiple participants and multiple viewpoints can be perplexing. Groups of reviewers see different aspects of a comparison of alternatives, and they can desire many comparisons of the measures of effectiveness or criteria. A comprehensive, objective evaluation needs to illustrate the various viewpoints of many constituents.

The transportation professional is faced with the challenge of presenting technical analyses to various groups in a manner permitting them to make their own decisions. The choice of a preferred alternative involves both quantitative aspects and intangibles or quality aspects. Analyses often seem to press in one direction while new viewpoints and other considerations can give a different perspective on the relative merits of the alternatives.

Presentation of technical information in understandable terms has become increasingly important in transportation studies. Professionals develop significant quantitative data to compare and contrast alternatives under investigation, and there is a need to render the results comprehensible for those making recommendations and those making decisions.

\section{Objectives}

At the same time, there is a need for the professional to produce a fair, objective analysis.

Because comparisons involve qualitative and quantitative data, the techniques used to make comparisons need to permit flexibility and adaptation to the various opinions and judgments.

- Describe an APPROACH for comparing alternatives

- Illustrate a matrix comparison PROCEDURE used in corridor studies

- Illustrate a PRESENTATION TECHNIQUE for review of complex data

The purposes of this paper are to present screening and comparison techniques used in the evaluation of corridor alternatives, to place one approach in perspective for others to consider, and to present a technique for comparing alternatives that has proven successful in recent endeavors. This paper draws upon case histories in corridor planning and preliminary route location to illustrate the technique, problems, and workable solutions. Recognition is given at this time to the significant contributions in those studies by other consultants and the many state departments of transportation involved.

Although development of the alternatives and their various measures are important elements of any study, this paper is limited to a discussion of the technique for comparing the alternatives. It does not address the development of the alternatives nor development of the data measures. The approach is believed to have applicability to other corridor studies, while recognition is given to the need to adapt the technique to the specific needs of a given project. 


\section{Approach for Comparison of Alternative Corridors}

There is considerable effort involved in the development of corridor alternatives and the various data descriptors for each. For the purposes of this presentation, those activities are assumed to be acceptably accurate, complete, and comprehensive. The "GIGO" rule still applies-Garbage In, Garbage Out!

Comparisons used to critique alternatives can involve a number of dislike measures, such as travel time saved and disruption of environmentally sensitive areas. The transportation professionals are in need of procedures to permit comparing these alternatives in some meaningful ways. Some procedure to give a ranking of the alternatives within any given measure of effectiveness becomes a necessity in most instances. Reviewers also desire to know how the alternatives might be ranked based on some grouping of the measures. At the same time, caution needs to be exercised to recognize when "double counting" of some measures is involved. ${ }^{1}$

Generally speaking, at least the following characteristics are desired for the procedure:

- Show relativity

- Be consistent in application

- Permit giving more important measures a greater weight or significance

- Be logical

- Be understandable to the participants

The overall approach described herein involves critically comparing a number of alternatives using a series of matrices. The context of the example case studies is a corridor feasibility study. Thus, the purpose of those activities in the case studies was to select the best corridor to use for feasibility analysis purposes. This places a high priority on finding an alternative with reasonably high benefits and low costs. In other applications, detailed consideration would be given to other possibilities, including a "no build" alternative.

The approach for this comparative analysis is to develop a data matrix to compare explicitly the alternatives under consideration. The technique used is such that the criteria measures can be given different weights, can be included or omitted in various comparison groupings, and can be used to rank the alternatives on more than one basis. This can be accomplished by developing several tables (matrixes) after definition of the study criteria and measures of effectiveness.

As applied in a number of recent studies, this technique was used to compare initial corridor alternatives and to make two sets of decisions. One involved a determination of additional corridors to be developed and to be tested. The second involved deciding upon additional measures of effectiveness to be used in the comparisons.

1. Material for this paper was taken from the corridor feasibility studies for ISTEA High Priority Corridor 18 and Corridor 20. HNTB Corporation was one of the key consultants in this work. The Departments of Transportation for the several states involved were Arkansas, Indiana, Kentucky, Louisiana, Michigan, Mississippi, Tennessee, and Texas. 


\section{Measures of Effectiveness}

Although the development of the criteria and measures of effectiveness is an interesting process, it is too broad a subject to include herein. However, those items used in a recent feasibility study are important to recognize as examples for reference later in this paper. The critique of each study alternative included a comparison of service, impacts, and costs.

Exhibit 1 summarizes some of the measures of effectiveness used in a recent feasibility study. These are grouped within the criteria established during the study for comparing and selecting alternatives. For example, one criteria was to improve traffic service for users of the facility (as compared with existing service), and six measures were selected to compare the alternatives.

The measures need to be good indicators for the criteria involved as well as being items which can be determined for all alternatives. One should also note that some measures can apply to more than one criteria and can lead to "double counting" or multiple counting in the follow-up tabulations.

For example, savings in vehicle operating costs apply to the criteria "improve traffic service for users" and "maximize economic benefits". Another less obvious example relates to minimizing

Exhibit 1: Example measures of effectiveness (corridor feasibility study)

\begin{tabular}{|c|c|c|}
\hline \multicolumn{2}{|r|}{ Criteria } & Measures of Effectiveness \\
\hline \multirow[t]{3}{*}{$\begin{array}{l}\text { 1. Service } \\
\text { Potential }\end{array}$} & $\begin{array}{l}\text { Maximize the population served directly } \\
\text { by the study corridor }\end{array}$ & Population served by the corridor \\
\hline & $\begin{array}{l}\text { Improve traffic service for users of the } \\
\text { facility }\end{array}$ & $\begin{array}{l}\text { Vehicle Kilometers of Travel (VKT) along the corridor } \\
\text { Savings in vehicle operating costs } \\
\text { Savings in travel time } \\
\text { Savings in accidents } \\
\text { Travel time for the full length of corridor } \\
\text { Vehicle Hours of Travel (VHT) on the corridor }\end{array}$ \\
\hline & $\begin{array}{l}\text { Maximize the traffic relief provided to } \\
\text { nearby transportation facilities }\end{array}$ & $\begin{array}{l}\text { VHT within a set study area } \\
\text { VKT within a set study area }\end{array}$ \\
\hline \multirow[t]{2}{*}{$\begin{array}{l}\text { 2. Potential } \\
\text { Impacts }\end{array}$} & Maximize Economic benefits & $\begin{array}{l}\text { Jobs added } \\
\text { Value added to goods produced } \\
\text { Wages added to job payrolls }\end{array}$ \\
\hline & Minimize Environmental impacts & $\begin{array}{l}\text { Area of wetlands taken for right of way } \\
\text { Water areas impacted } \\
\text { Area of forest land taken for right of way } \\
\text { Area of agriculture taken for right of way } \\
\text { Number of sites impacted relative to cemeteries, public } \\
\quad \text { parks, National forests, and Superfund sites } \\
\text { Length of new loop roads required } \\
\text { Length of route required on new rural location }\end{array}$ \\
\hline \multirow[t]{2}{*}{ 3. Costs } & $\begin{array}{l}\text { Maximize savings in costs to maintain } \\
\text { highways }\end{array}$ & Highway maintenance costs \\
\hline & Minimize development costs & $\begin{array}{l}\text { Construction costs } \\
\text { Right of Way costs } \\
\text { Mitigation costs }\end{array}$ \\
\hline
\end{tabular}


environmental impacts and costs. A measure for the environmental impact potential is "area of wetlands taken for right of way" while the cost of the wetlands is included in both the mitigation costs and in the construction costs.

\section{Comparisons Using Measures of Effectiveness}

Once criteria are designated for selection of a preferred alternative, measures of effectiveness can be established. These need to be ones that can actually be measured to a degree of acceptable accuracy within the study parameters. Once identified, data can be developed for each measure relative to each alternative under study.

The initial step in the comparison of alternatives is to develop a table summarizing the measures of effectiveness as established for each study alternative. Data in one study were grouped into four categories-socio-economic and environmental, traffic service, costs, and feasibility.

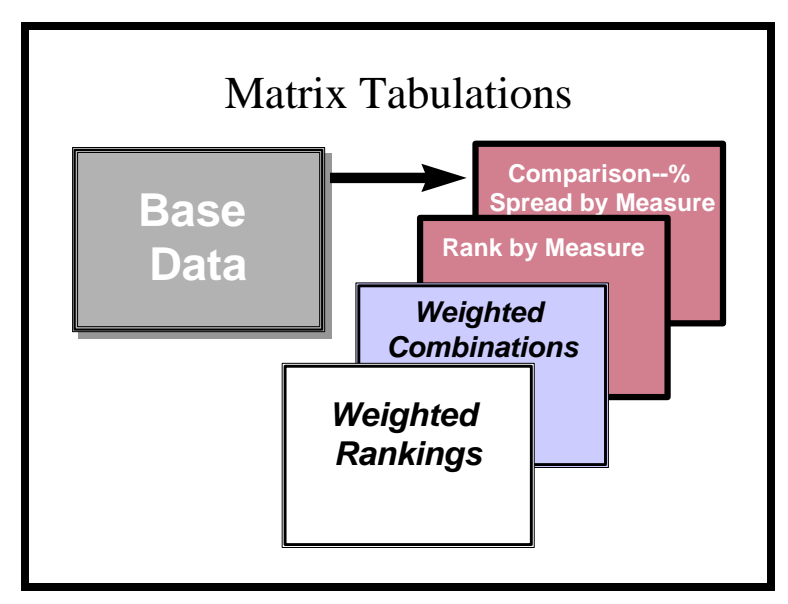

For brevity's sake, an excerpt of the full matrix of data is used for the remainder of this paper to illustrate the approach. Exhibit 2 presents a portion of the resulting tabulation of data for discussion purposes. Note that additional measures and ratios of measures can be used in the comparisons when deemed appropriate.

One of the first questions to be addressed is, "How do the alternatives "rank" for each measure of effectiveness?" To address this question, the review of each measure for all alternatives can be made easier if the data are placed on a common basis, say showing how each alternative's measure relates to the maximum value for that measure. For example, what percent of the traffic service provided by the best alternative is there provided by the other alternatives? This can be followed by a ranking of the alternatives, and a comparison for groups of measures.

Four matrix comparisons have been developed in the illustrative studies. The four tables are discussed in the following paragraphs. Excerpts of the four matrix tables are included as Exhibits 3 through 6. In brief, these provide for:

- Calculating the relative values for the alternatives within each measure of effectiveness

- Ranking of alternatives within each measure of effectiveness

- Calculating weighted values for various combinations of the measures of effectiveness

- Ranking of alternatives for combinations and groups of measures of effectiveness.

\section{Initial Comparisons}

The first step in the comparison process is to place each set of data for each measure on a common comparison basis. Experience has shown that the use of a percentage of the maximum value in each row (i.e. for each measure) produces a useful indicator and one that often is desired by the 
Exhibit 2: Example of raw data matrix (excerpt)

\begin{tabular}{|l|r|r|r|r|r|r|r|}
\hline \multicolumn{1}{|c|}{ Measure } & Alt 1 & Alt 2 & Alt 3 & Alt 4 & Alt 5 & \multicolumn{1}{c|}{ Alt 6 } & Alt 7 \\
\hline \hline Population served by route, millions & 2.79 & 3.01 & 2.99 & 3.02 & 3.22 & 3.47 & 3.52 \\
\hline Number jobs added & 9,700 & 9,200 & 9,500 & 10,400 & 11,000 & 11,900 & 12,700 \\
\hline Travel Time Savings, millions \$ & $\$ 747$ & $\$ 721$ & $\$ 757$ & $\$ 806$ & $\$ 852$ & $\$ 939$ & $\$ 1,006$ \\
\hline Accident Cost Reduction, millions $\$$ & $\$ 2,315$ & $\$ 2,524$ & $\$ 2,248$ & $\$ 2,567$ & $\$ 2,730$ & $\$ 3,134$ & $\$ 3,247$ \\
\hline Total Cost in millions & $\$ 3,192$ & $\$ 3,363$ & $\$ 3,359$ & $\$ 3,393$ & $\$ 3,599$ & $\$ 4,337$ & $\$ 4,832$ \\
\hline Travel Discounted B/C & 1.68 & 1.66 & 1.56 & 1.72 & 1.72 & 1.58 & 1.49 \\
\hline
\end{tabular}

Exhibit 3: Example calculation of the relative values for each measure as a percent of maximum raw data value

\begin{tabular}{|l|r|r|r|r|r|r|r|}
\hline \multicolumn{1}{|c|}{ Measure } & Alt 1 & \multicolumn{1}{c|}{ Alt 2 } & \multicolumn{1}{c|}{ Alt 3 } & \multicolumn{1}{c|}{ Alt 4 } & \multicolumn{1}{c|}{ Alt 5 } & \multicolumn{1}{c|}{ Alt 6 } & Alt 7 \\
\hline \hline Population served by route, millions & $79 \%$ & $86 \%$ & $85 \%$ & $86 \%$ & $91 \%$ & $99 \%$ & $100 \%$ \\
\hline Number jobs added & $76 \%$ & $72 \%$ & $75 \%$ & $82 \%$ & $87 \%$ & $94 \%$ & $100 \%$ \\
\hline Travel Time Savings, millions \$ & $74 \%$ & $72 \%$ & $75 \%$ & $80 \%$ & $85 \%$ & $93 \%$ & $100 \%$ \\
\hline Accident Cost Reduction, millions \$ & $71 \%$ & $78 \%$ & $69 \%$ & $79 \%$ & $84 \%$ & $97 \%$ & $100 \%$ \\
\hline Total Cost in millions & $66 \%$ & $70 \%$ & $70 \%$ & $70 \%$ & $74 \%$ & $90 \%$ & $100 \%$ \\
\hline Travel Discounted B/C & $98 \%$ & $97 \%$ & $91 \%$ & $100 \%$ & $100 \%$ & $92 \%$ & $87 \%$ \\
\hline
\end{tabular}

Exhibit 4: Example ranking of alternatives within each measure (5=best, 1=least desirable)

\begin{tabular}{|l|r|r|r|r|r|r|r|}
\hline \multicolumn{1}{|c|}{ Measure } & \multicolumn{1}{|c|}{ Alt 1 } & \multicolumn{1}{|c|}{ Alt 2 } & \multicolumn{1}{|c|}{ Alt 3 } & \multicolumn{1}{c|}{ Alt 4 } & \multicolumn{1}{c|}{ Alt 5 } & Alt 6 & \multicolumn{1}{c|}{ Alt 7 } \\
\hline \hline Population served by route, millions & 1.0 & 2.2 & 2.1 & 2.3 & 3.4 & 4.7 & 5.0 \\
\hline Number jobs added & 1.6 & 1.0 & 1.3 & 2.4 & 3.1 & 4.1 & 5.0 \\
\hline Travel Time Savings, millions \$ & 1.4 & 1.0 & 1.5 & 2.2 & 2.8 & 4.1 & 5.0 \\
\hline Accident Cost Reduction, millions \$ & 1.3 & 2.1 & 1.0 & 2.3 & 2.9 & 4.5 & 5.0 \\
\hline Total Cost, in millions & 5.0 & 4.6 & 4.6 & 4.5 & 4.0 & 2.2 & 1.0 \\
\hline Travel Discounted B/C & 4.3 & 4.0 & 2.2 & 5.0 & 5.0 & 2.6 & 1.0 \\
\hline
\end{tabular}

Exhibit 5: Example summation of weighted values

\begin{tabular}{|l|r|r|r|r|r|r|r|}
\hline \multicolumn{1}{|c|}{ Measure } & \multicolumn{1}{|c|}{ Alt 1 } & \multicolumn{1}{c|}{ Alt 2 } & \multicolumn{1}{c|}{ Alt 3 } & \multicolumn{1}{c|}{ Alt 4 } & Alt 5 & Alt 6 & Alt 7 \\
\hline \hline All factors, equal weight, 47 items & 139.1 & 170.6 & 130.1 & 184.4 & 190.2 & 146.4 & 120.0 \\
\hline Socio-Economic and Environmental (20 items) & 52.0 & 69.8 & 48.9 & 73.3 & 78.4 & 64.6 & 61.6 \\
\hline Socio-Economic and Environmental (15 items) & 40.4 & 51.8 & 39.2 & 54.7 & 57.7 & 49.1 & 45.1 \\
\hline Traffic Service (8 items) & 20.1 & 21.7 & 19.6 & 24.0 & 25.4 & 27.9 & 24.0 \\
\hline Development Cost (9 items) & 34.6 & 39.5 & 35.3 & 40.2 & 38.9 & 21.7 & 14.7 \\
\hline Development Cost (5 items) & 21.0 & 24.2 & 19.8 & 23.3 & 22.6 & 10.0 & 6.7 \\
\hline Feasibility (9 items) & 27.4 & 34.7 & 21.7 & 42.3 & 43.3 & 30.0 & 18.7 \\
\hline
\end{tabular}


Exhibit 6: Example ranking of alternatives for combinations and groups of measures (5=best, $1=$ least desirable)

\begin{tabular}{|l|r|r|r|r|r|r|r|}
\hline \multicolumn{1}{|c|}{ Measure } & \multicolumn{1}{|c|}{ Alt 1 } & \multicolumn{1}{c|}{ Alt 2 } & \multicolumn{1}{c|}{ Alt 3 } & Alt 4 & \multicolumn{1}{c|}{ Alt 5 } & Alt 6 & Alt 7 \\
\hline \hline All factors, equal weight (47 items) & 2.1 & 3.9 & 1.6 & 4.7 & 5.0 & 2.5 & 1.0 \\
\hline Socio-Economic and Environmental (20 items) & 1.4 & 3.8 & 1.0 & 4.3 & 5.0 & 3.1 & 2.7 \\
\hline Socio-Economic and Environmental (15 items) & 1.3 & 3.7 & 1.0 & 4.4 & 5.0 & 3.1 & 2.3 \\
\hline Traffic Service (8 items) & 1.2 & 2.0 & 1.0 & 3.1 & 3.8 & 5.0 & 3.1 \\
\hline Development Cost (9 items) & 4.1 & 4.9 & 4.2 & 5.0 & 4.8 & 2.1 & 1.0 \\
\hline Development Cost (5 items) & 4.3 & 5.0 & 4.0 & 4.8 & 4.6 & 1.8 & 1.0 \\
\hline Feasibility (9 items) & 2.4 & 3.6 & 1.5 & 4.8 & 5.0 & 2.8 & 1.0 \\
\hline
\end{tabular}

reviewers as well as by the analyst. Many are looking at the raw data and trying to determine which Alternative has the highest number, the lowest, and how the others relate to these extremes.

The first matrix (Exhibit 3) places the comparative data for the seven alternatives on a common base by converting the values for each measure to a percent of the maximum value for all alternatives. In reviewing this matrix, one can readily discern the alternative with the maximum value for each measure (shown as 100\%) as well as how compact or close together the measures are for all alternatives.

For example, the highest population served at 3.52 million (Alternative 7) is used as 100\%, while Alternative 1 with 2.79 million calculates to be $79 \%$ of that value. The least population served (Alternative 1) shows serving only $79 \%$ of that served by the best alternative from this single measure.

A reviewer can readily see how closely the measures are grouped or how far apart they are for the alternatives. [Note that Exhibit 3 is an excerpt of the full table developed in the case studies.]

High vs. Low Values, Which Is Best??

- There are different goals for individual measures. For example, -Population Served, result desired needs to be HIGH

-Cost to Build, result desired needs to be LOW
The second matrix (Exhibit 4) then ranks the alternatives (from 5 is best to 1 is least desirable) taking into consideration whether higher values are best or lower values are best. Thus, for the illustration used herein, a value of 5 is always given to the "best" alternative for that measure. The first matrix (Exhibit 4) clearly shows the spread and the grouping of alternatives within a given measure, while the second matrix (Exhibit 5) places all rankings on a common scale, 1 to 5 (or any other range desired).

In the example, for the measure "population

served", Alternative 7 is the best of the alternatives and is ranked 5. Alternative 1 has the lowest value and is ranked 1. Each of the other alternatives is pro-rated between 1 and 5 according to its relative value.

Note that in this example, the best Alternative for each criteria measure involved is ranked 5. The 
least desirable is ranked 1. Others are scaled (pro-rated) between 1 and 5. If several of the alternatives have data that are very close to the "best", they will receive rankings very close to a 5 . For example, in looking at the criteria measure for "Total Cost" in Exhibit 4, three alternatives have very close results near the least amount and are ranked 4.6, 4.6, and 4.5.

\section{Combinations of Measures}

All reviewers have questions about how the alternatives would be ranked when considering various combinations of the measures. The third matrix (Exhibit 5) presents a series of combinations of measures using data from the previous tables. For example, all 8 of the Traffic Service measures are combined with equal weight given to each measure by summing the rankings from the previous table for each alternative. Alternative 1

receives 20.1 ranking points; Alternative 2, 21.7; etc.

The fourth matrix (Exhibit 6) repeats the ranking procedure (from 5 is best to 1 is least desirable) for the combinations of measures. This process facilitates the comparison of the alternatives by showing for all measures the "best" alternative as a " 5 ". For example, with equal weighting for the 8 Traffic

Service measures, Alternative 6 has the highest total at 27.9 points in the third matrix and is considered the best overall. Alternative 3 has the lowest total (19.6) and is considered the least desirable. Each other alternative is ranked on a pro-rata basis between 1 and 5 .

\section{Variations in Ranking Procedures}

Any of several alternative ranking procedures could be used for Exhibits 4 and 6 . For example, ranking from 1 to 10 could be used instead of from 1 to 5, or a "1" could be used as being best instead of least desirable. In developing the combinations, different weights can be given to each measure in order to permit giving different importance to the various measures.

Exhibit 7: Some combinations of measures

\begin{tabular}{|c|l|}
\hline Group & \multicolumn{1}{|c|}{ Measures Included } \\
\hline \hline A & 3 State Economic Impact factors \\
\hline B & 3 Area Economic impact factors \\
\hline C & 2 Benefit Cost ratios (Travel and State) \\
\hline D & Socio-Eco-Env (SEE) factors + Benefit/Cost Ratio (B/C factors) \\
\hline E & Savings in Op Cost, Accidents, Travel Time (Savings Factors) \\
\hline F & Socio-Economic and Environmental (SEE) Factors \\
\hline G & SEE Factors + Savings factors + Cost/VKT + ADT/km \\
\hline H & 14 Distinguishing Measures \\
\hline J & SEE + 2 Traffic Service + Travel B/C \\
\hline
\end{tabular}

Another variation that was explored involves using what some termed a "constant yardstick" when developing the comparisons shown in Exhibit 3, Calculation of the Relative Value for Each Measure as a Percent of the Maximum Raw Data Value. This modified procedure relates each measure to the extremes in the range for all measures rather than to the extremes for each measure.

The tables presented herein 
were developed in a spreadsheet format with look-up values for the "best" and "least desirable" ranking numbers to be used. In addition, a separate look-up permits giving the different weights to the individual measures of effectiveness and/or to a set group of measures. For example, each of the measures within the socio-economic impact group can be given individual weights to increase the relative importance of each measure. Or the entire group can be given a weighting different from the other measures or groups of measures.

Exhibit 7 illustrates some of the 45 combinations of measures developed in one recent feasibility study to accommodate questions raised by reviewers and analysts on whether rankings would change if certain measures were deemed more important than others. Some groupings involved multiple counting of specific measures. These groupings are in addition to the examples in Exhibits 5 and 6 and are derived from the specific measures identified in Exhibit 1. In addition, specific groups were further modified to investigate placing more or less importance on specific measures or groups of measures. For example, the effect on rankings of doubling, tripling, etc. the weight given to "Total Cost" was investigated.

Does this technique seem overly complex? Is it simply too involved to comprehend? It has worked well with administrators and with technical (professional) staff. In recent corridor studies the use of this approach has proven very effective in permitting advisory committees to compare the alternatives realistically. For pubic presentations, simplified visual aids were desired to present the essence of the comparisons and rankings while making details available for those desiring more information. The initial step is to provide a clear explanation. Most people seemed to be more inter-

Comparison of Alternatives in Matrix Format

- Base Data for each Alternative with Measures of Effectiveness

- Comparison of Alternatives within each Measure of Effectiveness

- Relative Ranking of Alternatives within each Measure of Effectiveness

- Combinations of Measures

- Weighted Ranking of Alternatives ested in having an overview of the process and in making their position clear rather than in dwelling upon the details of the analyses.

\section{Conclusions}

Different individuals-whether professional or lay persons-often desire to explore the ranking of alternatives in various ways. They can give different importance to some measures of effectiveness (or criteria), or they may simply wonder how rankings might change for different viewpoints on the measures to be considered.

The approach for the comparative analyses presented herein is to develop several data matrices to compare explicitly the alternatives under consideration. The technique used is such that the criteria measures can be given different weights, can be included or omitted in various comparison groupings, and can be used to rank the alternatives on more than one basis.

One caution is to insure adequate reliability of data used in the matrices. Another is to recognize when there is double counting of measures and to insure that reviewers understand the significance of double counting data.

This type of ranking procedure is not a substitute for sound analysis and sound judgment. The calculations do not determine which alternative is the best; they simply present the results of the data 
input to the process in a manner facilitating analysis. Poor input yields poor results. If the resulting rankings do not support the most logical and defensible solution, then something is amiss and further evaluation is needed. 